Review

\title{
Application of p16/Ki-67 dual-staining cytology in cervical cancers
}

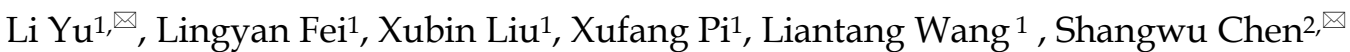 \\ 1. Department of Pathology, the First Affiliated Hospital, Sun Yat-sen University, Guangzhou 510080, People's Republic of China. \\ 2. State Key Laboratory for Biocontrol, Guangdong Key Laboratory of Pharmaceutical Functional Genes, Key Laboratory of Gene Engineering of the Ministry \\ of Education, Department of Biochemistry, School of Life Sciences, Sun Yat-sen University, Guangzhou 510275, People's Republic of China. \\ $\square$ Corresponding authors: Li Yu, Department of Pathology, the First Affiliated Hospital, Sun Yat-sen University, Guangzhou 510080, People's Republic of \\ China. Tel.: 86-20-87755766-8864, Fax: 86-20-87331780, E-mail: yuli5@mail.sysu.edu.cn; Shangwu Chen, Department of Biochemistry, School of Life Sciences, Sun \\ Yat-sen University, Guangzhou 510275, People's Republic of China, Tel.: 86-20-39332958, Fax: 86-20-39332950, E-mail: 1sschshw@mail.sysu.edu.cn. \\ (1) Ivyspring International Publisher. This is an open access article distributed under the terms of the Creative Commons Attribution (CC BY-NC) license \\ (https://creativecommons.org/licenses/by-nc/4.0/). See http://ivyspring.com/terms for full terms and conditions.
}

Received: 2019.01.02; Accepted: 2019.05.07; Published: 2019.06.02

\begin{abstract}
Cytology-based Papanicolaou test on and primary HPV screening have been widely used in the identification of cervical cancer and precancerous lesions, which is of great significance for the prevention and treatment of cervical cancer. Patients diagnosed as ASCUS/LSIL usually need follow-up because some of them may develop into $\mathrm{CIN} 2+$. The consequences of women positive for HPV vary from person to person; some of them may progress into cervical dysplasia, reversible forms of precancerous lesions, and eventually invasive cervical cancer. Therefore, it is necessary to establish an effective biomarker to triage different patients according to the preliminary screening results. p16 acts as a cell cycle regulatory protein that induces cell cycle arrest, and $\mathrm{Ki}-67$ is a cell proliferation marker. Under physiological conditions, they could not co-express in the same cervical epithelial cells. The co-expression of these two molecules suggests a deregulation of the cell cycle mediated by HR-HPV infection and predicts the presence of high-grade cervical epithelial lesions. There is increasing evidence that p16/Ki-67 dual-staining cytology can be used as an alternative biomarker, showing overall high sensitivity and specificity for identifying high-grade $\mathrm{CIN}$ and cervical cancer. In this review, we discuss the significance of p16/Ki-67 dual-staining and summarize its application in the screening and triaging of cervical cancer and precancerous lesions.
\end{abstract}

Key words: p16/Ki-67 dual-staining; cytology; HPV; cervical cancer screening; CIN

\section{Introduction}

Cervical cancer is the fourth most common malignant tumor among women worldwide [1], and the infection of human papillomavirus (HPV) is essential cause. Certain HPV types, in particular high-risk HPV (HR-HPV) 16 and 18, may lead to cervical dysplasia, reversible forms of precancerous lesions. Persistent infection of the cervix can cause irreversible changes leading to carcinoma in situ and eventually invasive cervical cancer. This results from the effects of HPV genes, particularly those encoding E6 and E7. Integration of HPV DNA into the host genome induces the expression of E6 and E7 [2]. E7 binds to and inactivates retinoblastoma protein (pRB), leading to the activation of the cell cycle [3]. E6 binds to and inactivates the p53 protein and deregulates the cell cycle in synergy with the inactivation of the cell cycle regulator pRB by E7. Expression of E7 and E6 can efficiently immortalize human primary keratinocytes and is required to induce and to maintain the transformed phenotype of cervical cancer cells [4].

Early diagnosis through various screening techniques is the main step in the prevention and treatment of cervical cancer. At present, three main methods, cytology, HPV testing, and cytology combined with HPV testing, have been applied to cervical cancer screening [5]. Papanicolaou test based on cytology (Pap test) is the earliest method for 
screening cervical cancer. It significantly reduces the morbidity and mortality caused by this cancer [6]. Cytology showed high specificity from $86 \%$ to $100 \%$, but provided relatively low sensitivity from $30 \%$ to $87 \%$ and subjectivity between observers $[7,8]$. The sensitivity of HPV testing to cervical cancer and its precursors is as high as $95 \%$. Therefore, detection of HR-HPV can be used as an alternative or auxiliary tool for cervical cancer screening to improve the sensitivity of cytology. HPV testing can prolong the screening interval, but the specificity is slightly lower than that of cytology $[7,9]$. The combination of HPV testing and cytology is expected to overcome their shortcomings [10-12].

Patients with mild cervical lesions diagnosed as low-grade squamous intraepithelial lesion (LSIL) and atypical squamous cells of undetermined significance (ASCUS) may progress to grade 2 or more severe cervical intraepithelial neoplasia (CIN2+) [13]. At this time, HPV DNA testing is introduced to triage minor abnormal cytology. Because of the low specificity of HPV DNA testing, many women will undergo colposcopy, especially among young women under 30 years of age with high HPV prevalence [14]. For example, the HR-HPV prevalence varies from $80 \%$ to $85 \%$ in women with LSIL. They are either directly referred to colposcopy or are followed up by cytology [14]. According to the FDA guidelines for primary HPV screening, women with positive HPV16 or HPV18 were immediately examined by colposcopy, while women with positive HPV but negative HPV16 or HPV18 were examined by cytology. If cytology is negative, they will be followed up 12 months later [15]. Many women with positive HPV need repeated cytological follow-up because of the relatively low sensitivity of cytology. Therefore, more effective markers are required to triage HPV-positive women with normal cytology or negative HPV16/18 and to identify women with potential high-grade CIN from cytological ASCUS/LSIL candidates. Accumulating evidence suggests that p16/Ki-67 dual-staining cytology is becoming an alternative biomarker, showing overall high sensitivity and specificity for identifying high-grade CIN [16-20]. This article will discuss the significance of p16/Ki-67 dual-staining and summarize its application in the screen and triage of cervical cancer and precancerous lesions.

\section{Significance of p16 and Ki-67 dual-staining cytology}

\subsection{Feature and function of p16}

p16INK4A (p16) is a tumor suppressor protein, also known as cyclin-dependent kinase inhibitor $2 \mathrm{~A}$ (CDKN2A) [21, 22]. It is encoded by the CDKN2A gene located on short arm of chromosome 9 (9p21.3) and named for its molecular weight and its role in inhibiting cyclin-dependent kinases 4 (CDK4) [23]. p16 can bind to CDK4 and CDK6, which plays an important role in the regulation of cell cycle. CDK4/6 normally forms a protein complex with cyclin D to phosphorylate $\mathrm{pRB}$. Upon phosphorylation, $\mathrm{pRB}$ disassociates from the transcription factor E2F1, leading to E2F1 translocation into the nucleus, where E2F1 induces the transcription of target genes that promote cell transition from G1 phase to $S$ phase. Therefore, p16 acts as a CDK inhibitor by preventing its interaction with cyclin $\mathrm{D}$, consequently prohibiting cell cycle progression [24, 25]. p16 downregulation can lead to cancer via the dysregulation of cell cycle progression. The p16 gene is frequently mutated in many cancers and downregulation of p16 expression is associated with increased risk of cancers [26, 27].

In cells with HPV infections, E7 protein competes for binding cell cycle regulatory proteins $\mathrm{pRb}$, resulting in the release of $\mathrm{E} 2 \mathrm{~F} 1$ from $\mathrm{pRb}$ and the activation of the cell cycle [4]. The disturbance of pRb-E2F1 pathway by E7 induces the overexpression and accumulation of p16 in the cells through a negative feedback loop [3, 28]. Strong and diffuse cytoplasmic and nuclear expression of p16 in cervical squamous cell carcinomas is predominantly associated with HR-HPV infection. Therefore, p16 is considered a surrogate marker for persistent HR-HPV infection and p16 overexpression has been observed in the majority of cervical precancers and cancers [29, 30].

\subsection{Feature and function of $\mathbf{K i}-67$}

Ki-67 is a cell proliferation marker, first defined by its city of origin (Kiel) and the number of original clone [31]. Ki-67 is a nuclear non-histone protein, which is encoded by MKI-67 gene and expressed in all phases of the cell cycle, except during the G0 phase [32]. Ki-67 exerts multiple functions in regulating cell cycle progression [33]. With the progression of cell cycle, it plays different roles, which is related to its distribution in the cells. Ki-67 is required for the normal cellular distribution and nucleolar association of heterochromatin during interphase [33]. During mitosis, Ki-67 is involved in the formation of the perichromosomal layer, which functions as a protective sheath around the chromosomes and provides a platform during nucleolar assembly, where Ki-67 serves as a biological surfactant to prevent the aggregation of mitotic chromosomes after nuclear envelope disassembly [34].

As a cell proliferation marker, $\mathrm{Ki}-67$ predicts the malignant potential of tumors. Immunohistochemical analysis of Ki-67 is usually performed on 
paraffin-embedded section and serves as an attractive index for prognosis and prediction in many tumors [31]. The detection of Ki-67 has been widely used in the auxiliary diagnosis of cervical precancers and cancers $[30,35]$.

\section{3 p 16/Ki-67 dual-staining cytology and its implication}

p16 is a tumor suppressor and $\mathrm{Ki}-67$ is a cellular marker for proliferation. The over-expression of p16 and expression of $\mathrm{Ki}-67$ under physiological situations are mutually exclusive and do not occur in the same cervical epithelial cell. Therefore, the p16/Ki-67 co-expression implies deregulation of the cell cycle induced by HR-HPV and detection of p16/Ki-67 co-expression can serve as a marker to predict the cell transformation by HR-HPV and the presence of high-grade CIN lesions [18, 19, 36]. Co-expression of p16/Ki-67 can be detected with antibodies against p16 and Ki-67. The brown cytoplasm/nuclear signal was displayed by p16 staining alone, and the red nuclear signal was displayed by Ki-67 staining alone. Positive p16/Ki-67 dual-staining cells had brown cytoplasm signals for p16 expression, and dark red to red brown nuclear signals reflected the co-localization of p16 and Ki-67 in the same cell (Fig 1). Slides with one or more cervical epithelial cells that were simultaneously stained for p16 and Ki-67 were classified as positive regardless of the morphological appearance of the cells [37].

The positive p16/Ki-67 dual-staining is associated with HR-HPV infection, particularly with HPV 16 and 18 [37-39]. The p16/Ki-67 positive rate in the HPV-positive women was $78.9 \%$, significantly higher than 9.4\% in the HPV-negative patients [39]. The association of p16/Ki-67 positivity with HPV16 and/or 18 infections was 2-4 fold stronger compared to the cases infected with other HR-HPV types [37, 38]. The positivity of $\mathrm{p} 16 / \mathrm{Ki}-67$ dual-staining also strongly indicates CIN2+ or high-grade squamous intraepithelial lesion (HSIL). The positive rates of p16/Ki-67 dual-staining in HR-HPV positive women with diagnoses of negative for intraepithelial lesion or malignancy (NILM), ASCUS, LSIL, atypical squamous cells cannot exclude HSIL (ASC-H), and HSIL were $3.0 \%, 23.6 \%, 25.8 \%, 78.6 \%$, and $100.0 \%$, respectively [40]. The positive rate increased from $31 \%$ in women with negative cytology to $92 \%$ in women with HSIL [41]. Similarly, the positive rate of p16/Ki-67 in women with CIN3 was $86 \%$, which was significantly higher than $24 \%$ in women without biopsy results (Table 1) [41]. All patients with cervical cancer showed double staining positive for P16/Ki-67. Other studies further confirmed that the positive rate of $\mathrm{p} 16 / \mathrm{Ki}-67$ increased significantly with the severity of cytological and histological abnormalities [19, 37, 38, 42]. The sensitivity and specificity of p16/Ki-67 dual-staining for CIN2+ were $74.9 \%-90.9 \%$ and $72.1 \%-95.2 \%$, respectively $[19,37,42$, 43]. The positive rate of CIN2+ detected by $\mathrm{p} 16 / \mathrm{Ki}-67$ dual-staining was $92.7 \%$, which was more sensitive than $71.1 \%$ by HPV16/18 genotyping alone [37]. Compared with HPV detection, p16/Ki-67 dual-staining has higher specificity in detecting CIN2+ and can significantly reduce the number of patients referred to colposcopy, especially for young women with high HPV infection rate $[19,42]$.

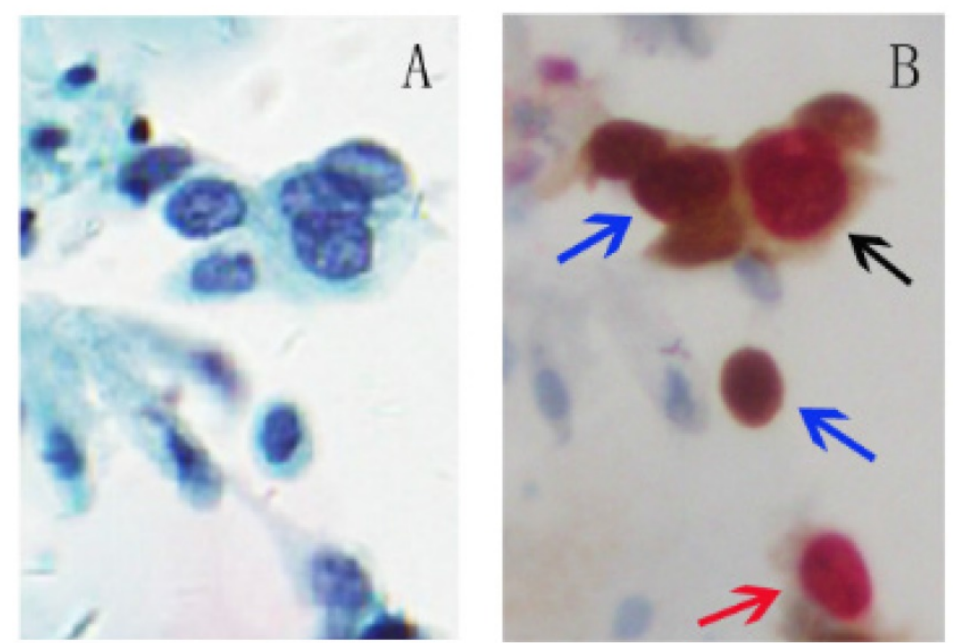

Fig 1. p16/Ki-67 dual-staining positive cells with morphological features of HSIL. A, Liquid-based cytology (SurePath). B, Slide A was dual-stained with p16/Ki-67. Cell with p16 staining alone (blue arrow) is characterized by a brown cytoplasmic/nuclear signal and cell with Ki-67 staining alone (red arrow) is presented in red nuclear signal. The positive p16/Ki-67 dual-staining cells (dark arrow) are characterized by a brown cytoplasmic signal for p16 overexpression and a dark red nuclear signal for p16/Ki-67 co-expression in the same cell. (45-year-old woman, CIN3, HPV16+, p16/Ki-67+). 


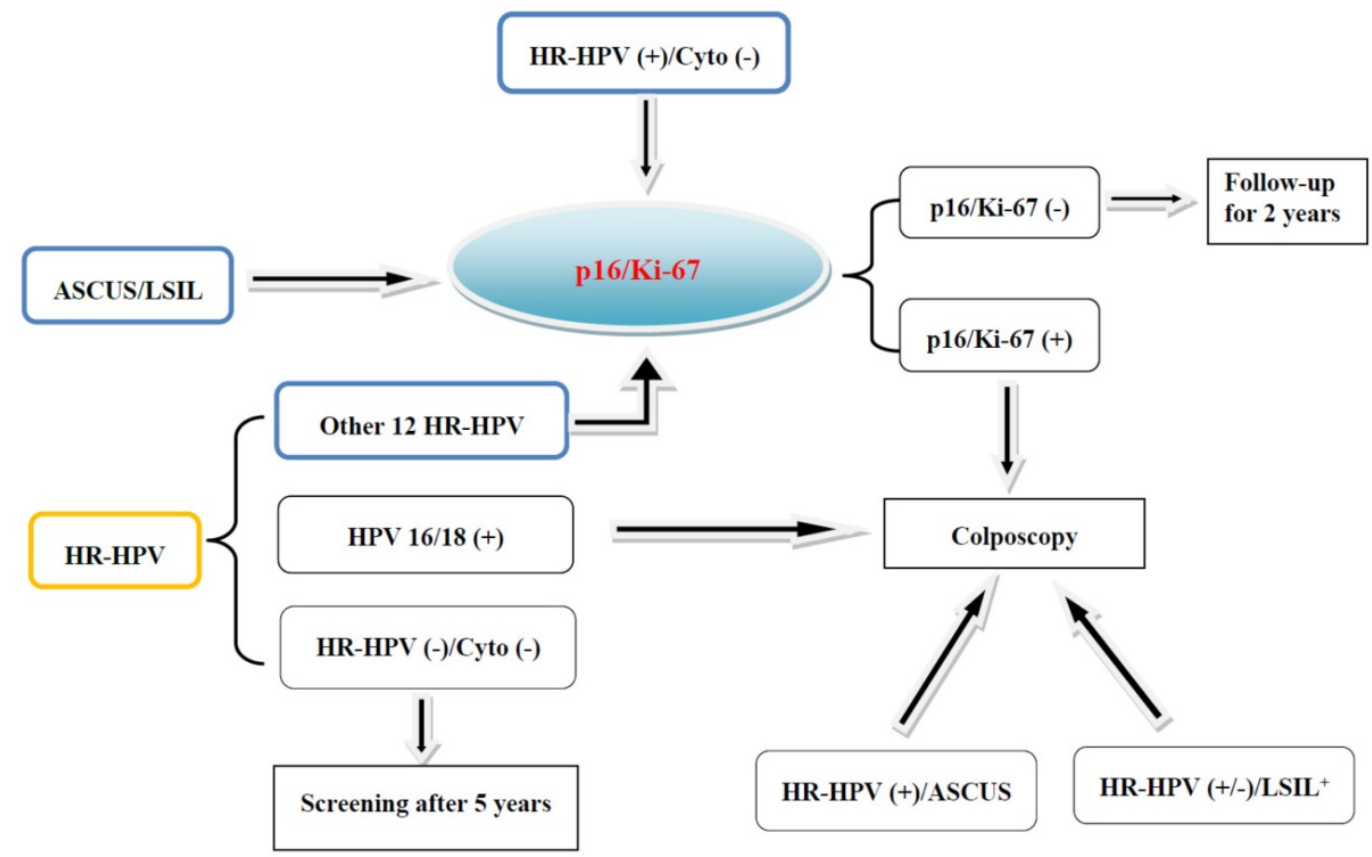

Fig 2. Screening and triaging of cervical cancer and the application of p16/Ki-67 dual-staining. The women diagnosed as ASCUS/LSIL, or positive for HR-HPV and free of cytological abnormalities, or positive for other 12 types of HR-HPV and negative for HPV 16 and 18 are recommended for the triage by p $16 / \mathrm{Ki}$-67 dual-staining.

\section{Application of p16/Ki-67 dual-staining}

\subsection{Triage of HPV-positive women}

The results of HPV infection vary greatly, and follow-up of HPV-positive women is complicated and laborious. To eliminate the unnecessary follow-up, HPV-positive women need a timely triage [10, 11]. Cytology is usually used to triage HPV-positive women who are negative for HPV16 or 18. Increasing studies have shown that p16/Ki-67 dual-staining is useful for the triage of HPV-positive women (Fig 2). The sensitivity of p16/Ki-67 dual-staining for triaging HPV-positive women was $74.9 \%$, significantly higher than $51.9 \%$ in Pap cytology, whereas the specificity was comparable [43]. During cytological screening, colposcopy is usually recommended for women with higher precancerous risk than the threshold $[13,14]$. It has been confirmed in the HPV-positive individuals that the precancerous risk of women with positive p16/Ki-67 dual-staining is much higher than the cytological risk threshold, while the risk of women with negative dual-staining is lower, which further indicates the potential role of dual-staining assay for the triage of HPV-positive women [41].

About 3-7\% women with normal Pap cytology and HR-HPV positive still suffer from high-grade CIN [44-46]. It was reported that p16/Ki-67 dual-staining can be used to triage these patients, and $25.4 \%$ of 425 women showed positive. The sensitivity for the detection of CIN2+ and CIN3+ was 91.9\% (34/37) and $96.4 \%(27 / 28)$, respectively, while the specificity was $82.1 \%$ and $76.9 \%$ [18]. Similar results can be replicated, and the sensitivity for CIN2+ was significantly higher than HPV16/18 genotyping (Table 1) [47].

Once the HPV-positive women with normal cytology were followed up, the 5-year cumulative incidence risks (CIR) for CIN2+ and CIN3+ were $12.2 \%$ and $6.9 \%$, respectively. Even if HPV16/18 genotyping was negative, the 5-year CIR for CIN3+ was still $3.6 \%$. If these women were negative for p16/Ki-67, the 5-year CIR for CIN3+ decreased to $3.3 \%$ [47]. Therefore, p16/Ki-67 dual-staining is suitable for triaging HPV-positive women with normal cytology to colposcopy to identify patients with a high probability of potential CIN2+ $[18,47]$.

\subsection{Triage of LSIL/ASCUS cytology}

Women diagnosed as ASCUS and LSIL have a $2.6 \%$ and 5.2\% 5-year risk for CIN3+, respectively [13]. Therefore, it is essential to identify potential HSIL from ASCUS/LSIL. The efficiency of p16/Ki-67 dual-staining has been evaluated and confirmed [17, 37, 39, 48, 49]. For example, the specificity of p16/Ki-67 dual-staining for detection of CIN3 was $75.2 \%$, significantly higher than $40.4 \%$ of HPV testing, although the sensitivity in the former was slightly lower [49]. Several other studies supported that p16/Ki-67 dual-staining had higher specificity and comparable sensitivity than HPV testing [17, 39, 48, 49]. p16/Ki-67 dual-staining also presented high positive predictive value for high-grade CIN, especially in women under 30 years of age, which can reduce the number of unnecessary colposcopy referrals $[37,39,48]$. 
Table 1. The value of p16/Ki-67 dual-staining in the triage of high-grade squamous intraepithelial lesion and its comparison with HPV testing and cytology

\begin{tabular}{|c|c|c|c|c|c|c|c|c|c|c|c|c|c|}
\hline \multirow[t]{2}{*}{ Studies } & \multirow[t]{2}{*}{ Subjects* } & \multicolumn{3}{|c|}{ Sensitivity \% } & \multicolumn{3}{|c|}{ Specificity \% } & \multicolumn{3}{|c|}{ PPV \% } & \multicolumn{3}{|c|}{ NPV \% } \\
\hline & & Dual & Cyto & HPV & Dual & Cyto & $\mathrm{HPV}$ & Dual & Cyto & $\mathrm{HPV}$ & Dual & Cyto & HPV \\
\hline \multirow[t]{2}{*}{ Ikenberg et al. 2013} & 181 CIN2+/25577 screening & 86.7 & 68.5 & 93.3 & 95.2 & 95.4 & 93.0 & 15.6 & 13.3 & 9.3 & 99.9 & 99.7 & 99.9 \\
\hline & $100 \mathrm{CIN} 3+/ 25577$ screening & 87.4 & 73.6 & 96.2 & 94.8 & 95.1 & 92.7 & & & & & & \\
\hline \multirow[t]{4}{*}{ Wentzensen et al. 2015} & $175 \mathrm{CIN} 2+/ 1509 \mathrm{HPV}+$ & 83.4 & 76.6 & & 58.9 & 49.6 & & 21.0 & 16.6 & & 96.4 & 94.2 & \\
\hline & 99 CIN3+/1509 HPV+ & 86.9 & 83.8 & & 56.9 & 48.7 & & 12.4 & 10.3 & & 98.4 & 97.7 & \\
\hline & $41 \mathrm{CIN} 2+/ 703 \mathrm{HPV}+$, Cyto- & 70.7 & & & 70.8 & & & 13.1 & & & 97.5 & & \\
\hline & 16 CIN3+/703 HPV+, Cyto- & 81.3 & & & 69.6 & & & 5.9 & & & 99.4 & & \\
\hline \multirow[t]{6}{*}{ Yu et al. 2016} & $20 \mathrm{CIN} 2+/ 1079$ screening & 75.0 & 65.0 & 100.0 & 79.5 & 76.2 & 76.9 & 6.5 & 4.9 & 7.5 & 99.4 & 99.1 & 100.0 \\
\hline & $6 \mathrm{CIN} 3+/ 1079$ screening & 83.3 & 83.3 & 100.0 & 78.8 & 75.8 & 75.9 & 2.2 & 1.9 & 2.3 & 99.9 & 99.9 & 100.0 \\
\hline & $218 \mathrm{CIN} 2+/ 463 \mathrm{HPV}+$ & 92.7 & 94.5 & & 52.7 & 53.5 & & 63.5 & 64.4 & & 89.0 & 91.6 & \\
\hline & $178 \mathrm{CIN} 3+/ 463 \mathrm{HPV}+$ & 95.0 & 98.3 & & 47.7 & 49.1 & & 53.1 & 54.7 & & 93.8 & 97.9 & \\
\hline & 48 CIN2+/256 ASCUS, LSIL & 87.5 & & 91.7 & 66.4 & & 55.8 & 37.5 & & 32.4 & 95.8 & & 96.7 \\
\hline & CIN3+/256 ASCUS, LSIL & 89.7 & & 89.7 & 62.1 & & 51.5 & 23.2 & & 19.1 & 97,9 & & 97.5 \\
\hline \multirow[t]{2}{*}{ Wright et al. 2017} & 367 CIN2+/3467 HPV+ & 70.3 & 51.8 & & 75.6 & 76.1 & & 26.2 & 21.1 & & 95.4 & 92.7 & \\
\hline & $243 \mathrm{CIN} 3+/ 3467 \mathrm{HPV}+$ & 74.9 & 51.9 & & 74.1 & 75.0 & & 18.5 & 14.0 & & 97.4 & 95.2 & \\
\hline \multirow[t]{2}{*}{ Tay et al. 2017} & 63 CIN2+/97 Cyto+ & 93.7 & & 85.7 & 76.5 & & 14.7 & 88.1 & & 65.1 & 86.7 & & 35.7 \\
\hline & 14 CIN2+/44 ASCUS, LSIL & 92.9 & & 85.7 & 76.7 & & 16.7 & 65.0 & & 32.4 & 95.8 & & 71.4 \\
\hline \multirow[t]{4}{*}{ Schmidt et al. 2011} & 77 CIN2+/361 ASCUS & 92.2 & & 90.9 & 80.6 & & 36.3 & & & & & & \\
\hline & 51 CIN3+ /361 ASCUS & 92.2 & & 90.2 & 80.6 & & 36.3 & & & & & & \\
\hline & 137 CIN2+/415 LSIL & 94.2 & & 96.4 & 68.0 & & 19.1 & & & & & & \\
\hline & 72 CIN3+/415 LSIL & 95.8 & & 95.8 & 68.0 & & 19.1 & & & & & & \\
\hline \multirow[t]{2}{*}{ Uijterwaal et al. 2014} & 58 CIN2+/256 ASC, LSIL, ASC-H, AGC & 89.7 & & 96.6 & 73.1 & & 68.1 & 54.7 & & 52.3 & 95.1 & & 98.2 \\
\hline & 27 CIN3+/256 ASC, LSIL, ASC-H, AGC & 100.0 & & 96.3 & 64.4 & & 57.6 & 28.4 & & 24.3 & 100.0 & & 99.1 \\
\hline \multirow[t]{4}{*}{ Bergeron et al. 2015} & 18 CIN2+/427 ASCUS & 94.4 & & 100.0 & 78.7 & & 60.4 & 16.3 & & 10.0 & 99.7 & & 100.0 \\
\hline & 14 CIN3+/427 ASCUS & 100.0 & & 100.0 & 78.2 & & 59.8 & 13.5 & & 7.8 & 100.0 & & 100.0 \\
\hline & 63 CIN2+/384 LSIL & 85.7 & & 98.4 & 53.3 & & 15.6 & 26.5 & & 18.6 & 95.0 & & 98.0 \\
\hline & 25 CIN3+/384 LSIL & 88.0 & & 100.0 & 49.3 & & 14.2 & 10.8 & & 7.5 & 98.3 & & 100.0 \\
\hline \multirow[t]{6}{*}{ White et al. 2016} & 138 CIN2+/471 ASCUS, LSIL & 75.4 & & 92.8 & 88.3 & & 48.9 & & & & & & \\
\hline & 48 CIN3+/471 ASCUS, LSIL & 79.2 & & 95.8 & 75.2 & & 40.4 & 26.6 & & 15.4 & 97.0 & & 99.8 \\
\hline & CIN2+/206 ASCUS & 71.9 & & 94.7 & 87.9 & & 64.4 & & & & & & \\
\hline & CIN3+/206 ASCUS & 71.4 & & 100.0 & 78.7 & & 56.9 & 17.8 & & 14.8 & 96.5 & & 100.0 \\
\hline & CIN2+/265 LSIL & 77.8 & & 91.4 & 88.6 & & 35.3 & & & & & & \\
\hline & CIN3+/265 LSIL & 85.7 & & 94.5 & 72.7 & & 28.9 & 30.6 & & 15.7 & 97.3 & & 97.3 \\
\hline \multirow[t]{2}{*}{ Petry et al. 2011} & 37 CIN2+/425 HPV+, Cyto- & 91.9 & & & 82.1 & & & & & & & & \\
\hline & $28 \mathrm{CIN} 3+/ 425 \mathrm{HPV}+$, Cyto- & 96.4 & & & 76.9 & & & & & & & & \\
\hline \multirow[t]{2}{*}{ Uijterwaal et al. 2015} & 48 CIN2+/762 HPV+, Cyto- & 68.8 & & & 72.8 & & & 25.2 & & & 94.6 & & \\
\hline & 15 CIN3+/762 HPV+, Cyto- & 73.3 & & & 70.0 & & & 8.7 & & & 98.5 & & \\
\hline \multirow[t]{3}{*}{ Ordi et al. 2014} & 378 HSIL, 18 CC/1123 Colposcopy & 90.9 & & 96.0 & 72.1 & & 41.4 & 63.9 & & 47.1 & 93.6 & & 94.9 \\
\hline & HSIL, CC/543 HSIL with Pap & 94.5 & & 96.1 & 73.4 & & 51.9 & 81.3 & & 71.7 & 91.6 & & 91.0 \\
\hline & HSIL, CC/580 ASC, AGC, LSIL, HPV+ & 88.9 & & 95.6 & 72.9 & & 36.7 & 37.4 & & 21.7 & 97.3 & & 97.8 \\
\hline
\end{tabular}

*The application of p16/Ki-67 dual-staining (Dual) in the triage of high-grade squamous intraepithelial lesion was compared with HPV testing (HPV) and cytology (Cyto) from four aspects: sensitivity, specificity, positive predictive value (PPV), and negative predictive value (NPV). In order to provide more valuable and comparable information, detailed sample data were shown in the table. The information of total subjects under screening or with a specific characteristic was presented after slash and the confirmed subjects with high-grade squamous intraepithelial lesion were shown before slash. AGC, atypical glandular cells; ASC, atypical squamous cells; ASC-H, atypical squamous cells cannot exclude HSIL; ASCUS, atypical squamous cells of undetermined significance; CC, cervical cancer; CIN, cervical intraepithelial neoplasia; HSIL, high-grade squamous intraepithelial lesion; LSIL, low-grade squamous intraepithelial lesion.

The absolute risk of CIN3 in women positive for HPV DNA alone was 15.6\% [49]. Among women with positive p16/Ki-67 and HPV DNA, the risk increased to $27 \%$, while among women with negative p16/Ki-67 and HPV DNA, the risk decreased to $1.2 \%$ [49]. Obviously, the combination of HPV DNA testing and p16/Ki-67 dual-staining can more effectively identify potential HSIL individuals with only slight cytological abnormalities.

\subsection{Monitoring recurrence and auxiliary diagnosis}

The combination of p16/Ki-67 dual-staining and HR-HPV detection can also be used to monitor the recurrence of CIN2+ (rCIN2+). Long-term follow-up studies showed that some patients treated for CIN2 and CIN3 may relapse and need to be closely monitored [50, 51]. Cytological and HR-HPV co-detection has recently been recommended as a follow-up strategy for these patients to avoid loss of potential HR-HPV negative CIN2+ lesions [50, 52]. However, the specificity of cytological detection or combined detection of cytology and HPV is limited. The sensitivity and specificity of cytology, HR-HPV detection, and p16/Ki-67 dual-staining for rCIN2+ in 364 women treated for CIN2/3 were analyzed. Their sensitivities were $82.1 \%, 84.6 \%$, and $69.2 \%$, respectively, but the specificity of $\mathrm{p} 16 / \mathrm{Ki}-67$ was $90.4 \%$, which was significantly higher than $70.8 \%$ in cytology and $76.2 \%$ in HR-HPV testing. The sensitivity of combined detection of p16/Ki-67 and HR-HPV was similar to cytology/HR-HPV co-detection $(87.2 \%$ vs. $89.7 \%)$, but the specificity was significantly improved $(74.2 \%$ vs. $58.1 \%)$, which resulted in higher positive predictive value and fewer referrals for colposcopy [53]. 
Cytological diagnosis of cervical glandular lesions is often difficult because of the difficulty in distinguishing inflammatory or hyperplastic changes from neoplasia [54]. In 40 cases of cervical adenocarcinoma, $92.5 \%$ of p16/Ki-67 dual-staining was positive, and only 1 of 16 cervical tissues without glandular lesions was dual-staining positive, suggesting that $\mathrm{p} 16 / \mathrm{Ki}-67$ dual-staining is a potential tool for the diagnosis of cervical glandular lesions [55].

\section{Conclusions}

p16/Ki-67 dual-staining cytology is of great significance in screening and triaging of cervical cancer and precancerous lesions. It provides a good risk marker for the stratification of HPV-positive women, including normal cytological patients, and for the identification of high-grade CIN from women diagnosed as ASCUS or LSIL. Compared with Pap cytology and HPV detection, it has higher sensitivity and specificity in detecting cervical precancerous lesions and cervical cancer. This is also helpful for the diagnosis of cervical glandular lesions. The combination of p16/Ki-67 dual-staining and HP-HPV detection is recommended as a strategy for monitoring women treated for high-grade CIN. We note that in some cases, it is difficult to judge the results of p16/Ki-67 dual-staining cytology [39, 47, 49, 56]. The morphological or automated evaluation of p16/Ki-67 dual-staining was also attempted [42, 57, 58]. It is believed that p16/Ki-67 dual-staining cytology has broad application prospects in the diagnosis and treatment of cervical cancer.

\section{Acknowledgments}

This study was funded by the National Natural Science Foundation of China (No. 31670788 and No. 81172485), the Ph.D. Program Foundation of Ministry of Education of China (No. 20130171110007), and Open Fund of Guangdong Key Laboratory of Pharmaceutical Functional Genes (No. 2014B030301 028 and No. 2017B030314021).

\section{Competing Interests}

The authors have declared that no competing interest exists.

\section{References}

1. Venkatesulu BP, Mallick S, Rath GK. Patterns of care of cervical cancer in the elderly: A qualitative literature review. J Geriatr Oncol. 2017; 8: 108-16.

2. Klaes R, Woerner SM, Ridder R, Wentzensen N, Duerst M, Schneider A, et al. Detection of high-risk cervical intraepithelial neoplasia and cervical cancer by amplification of transcripts derived from integrated papillomavirus oncogenes. Cancer Res. 1999; 59: 6132-6.

3. Klaes R, Benner A, Friedrich T, Ridder R, Herrington S, Jenkins D, et al. p16INK4a immunohistochemistry improves interobserver agreement in the diagnosis of cervical intraepithelial neoplasia. Am J Surg Pathol. 2002; 26: 1389-99.
4. Pauck A, Lener B, Hoell M, Kaiser A, Kaufmann AM, Zwerschke W, et al. Depletion of the cdk inhibitor p16INK4a differentially affects proliferation of established cervical carcinoma cells. J Virol. 2014; 88: 5256-62.

5. Wentzensen N, Schiffman M. Filling a gap in cervical cancer screening programmes. Lancet Oncol. 2014; 15: 249-51.

6. Arbyn M, Raifu AO, Weiderpass E, Bray F, Anttila A. Trends of cervical cancer mortality in the member states of the European Union. Eur J Cancer. 2009; 45: 2640-8.

7. Cuzick J, Clavel C, Petry KU, Meijer CJ, Hoyer H, Ratnam S, et al. Overview of the European and North American studies on HPV testing in primary cervical cancer screening. Int J Cancer. 2006; 119: 1095-101.

8. Nanda K, McCrory DC, Myers ER, Bastian LA, Hasselblad V, Hickey JD, et al. Accuracy of the Papanicolaou test in screening for and follow-up of cervical cytologic abnormalities: a systematic review. Ann Intern Med. 2000; 132: 810-9.

9. Ordi J, Alonso I, Torne A, Esteve R, Sierra E, Campo E, et al. Human papillomavirus load in Hybrid Capture II assay: does increasing the cutoff improve the test? Gynecol Oncol. 2005; 99: 313-9.

10. Castle PE, Sideri M, Jeronimo J, Solomon D, Schiffman M. Risk assessment to guide the prevention of cervical cancer. J Low Genit Tract Dis. 2008; 12: 1-7.

11. Rijkaart DC, Berkhof J, van Kemenade FJ, Coupe VM, Hesselink AT, Rozendaal L, et al. Evaluation of 14 triage strategies for HPV DNA-positive women in population-based cervical screening. Int J Cancer. 2012; 130: 602-10.

12. Dijkstra MG, van Niekerk D, Rijkaart DC, van Kemenade FJ, Heideman DA, Snijders PJ, et al. Primary hrHPV DNA testing in cervical cancer screening: how to manage screen-positive women? A POBASCAM trial substudy. Cancer Epidemiol Biomarkers Prev. 2014; 23: 55-63.

13. Katki HA, Schiffman M, Castle PE, Fetterman B, Poitras NE, Lorey T, et al. Benchmarking CIN $3+$ risk as the basis for incorporating HPV and Pap cotesting into cervical screening and management guidelines. J Low Genit Tract Dis. 2013; 17: S28-35.

14. Massad LS, Einstein MH, Huh WK, Katki HA, Kinney WK, Schiffman M, et al. 2012 updated consensus guidelines for the management of abnormal cervical cancer screening tests and cancer precursors. Obstet Gynecol. 2013; 121: 829-46.

15. Wright TC, Stoler MH, Behrens CM, Sharma A, Zhang G, Wright TL. Primary cervical cancer screening with human papillomavirus: end of study results from the ATHENA study using HPV as the first-line screening test. Gynecol Oncol. 2015; 136: 189-97.

16. Wentzensen N, Schwartz L, Zuna RE, Smith K, Mathews C, Gold MA, et al. Performance of p16/Ki-67 immunostaining to detect cervical cancer precursors in a colposcopy referral population. Clin Cancer Res. 2012; 18: 4154-62.

17. Schmidt D, Bergeron C, Denton KJ, Ridder R, European CCSG. p16/ki-67 dual-stain cytology in the triage of ASCUS and LSIL papanicolaou cytology: results from the European equivocal or mildly abnormal Papanicolaou cytology study. Cancer Cytopathol. 2011; 119: 158-66.

18. Petry KU, Schmidt D, Scherbring S, Luyten A, Reinecke-Luthge A, Bergeron $\mathrm{C}$, et al. Triaging Pap cytology negative, HPV positive cervical cancer screening results with p16/Ki-67 Dual-stained cytology. Gynecol Oncol. 2011; 121: 505-9.

19. Ikenberg H, Bergeron C, Schmidt D, Griesser H, Alameda F, Angeloni C, et al. Screening for cervical cancer precursors with p16/Ki-67 dual-stained cytology: results of the PALMS study. J Natl Cancer Inst. 2013; 105: 1550-7.

20. Tay TKY, Lim KL, Hilmy MH, Thike AA, Goh ST, Song LH, et al. Comparison of the sensitivity and specificity of p16/Ki-67 dual staining and HPV DNA testing of abnormal cervical cytology in the detection of histology proven cervical intraepithelial neoplasia grade 2 and above (CIN 2+). Malays J Pathol. 2017; 39: 257-65.

21. von Knebel Doeberitz M. New markers for cervical dysplasia to visualise the genomic chaos created by aberrant oncogenic papillomavirus infections. Eur J Cancer. 2002; 38: 2229-42.

22. Cuschieri K, Wentzensen N. Human papillomavirus mRNA and p16 detection as biomarkers for the improved diagnosis of cervical neoplasia. Cancer Epidemiol Biomarkers Prev. 2008; 17: 2536-45.

23. Serrano M, Hannon GJ, Beach D. A new regulatory motif in cell-cycle control causing specific inhibition of cyclin D/CDK4. Nature. 1993; 366: 704-7.

24. Kalof AN, Cooper K. p16INK4a immunoexpression: surrogate marker of high-risk HPV and high-grade cervical intraepithelial neoplasia. Adv Anat Pathol. 2006; 13: 190-4.

25. Cicenas J, Kvederaviciute $\mathrm{K}$, Meskinyte I, Meskinyte-Kausiliene E, Skeberdyte A, Cicenas J. KRAS, TP53, CDKN2A, SMAD4, BRCA1, and BRCA2 Mutations in Pancreatic Cancer. Cancers (Basel). 2017; 9; 42

26. Leversha MA, Fielding P, Watson S, Gosney JR, Field JK. Expression of p53, pRB, and p16 in lung tumours: a validation study on tissue microarrays. J Pathol. 2003; 200: 610-9.

27. Makitie AA, MacMillan C, Ho J, Shi W, Lee A, O'Sullivan B, et al. Loss of p16 expression has prognostic significance in human nasopharyngeal carcinoma. Clin Cancer Res. 2003; 9: 2177-84.

28. Khleif SN, DeGregori J, Yee CL, Otterson GA, Kaye FJ, Nevins JR, et al. Inhibition of cyclin D-CDK4/CDK6 activity is associated with an E2F-mediated induction of cyclin kinase inhibitor activity. Proc Natl Acad Sci US A. 1996; 93: 4350-4. 
29. von Keyserling H, Kuhn W, Schneider A, Bergmann T, Kaufmann AM. p16INK(4)a and p14ARF mRNA expression in Pap smears is age-related. Mod Pathol. 2012; 25: 465-70.

30. Yu L, Wang L, Zhong J, Chen S. Diagnostic value of p16INK4A, Ki-67, and human papillomavirus L1 capsid protein immunochemical staining on cell blocks from residual liquid-based gynecologic cytology specimens. Cancer Cytopathol. 2010; 118: 47-55.

31. Menon SS, Guruvayoorappan C, Sakthivel KM, Rasmi RR. Ki-67 protein as a tumour proliferation marker. Clin Chim Acta. 2019; 491: 39-45.

32. Schluter C, Duchrow M, Wohlenberg C, Becker MH, Key G, Flad HD, et al. The cell proliferation-associated antigen of antibody Ki-67: a very large, ubiquitous nuclear protein with numerous repeated elements, representing a new kind of cell cycle-maintaining proteins. J Cell Biol. 1993; 123: 513-22.

33. Sun X, Kaufman PD. Ki-67: more than a proliferation marker. Chromosoma. 2018; 127: 175-86.

34. Cuylen S, Blaukopf C, Politi AZ, Muller-Reichert T, Neumann B, Poser I, et al. Ki-67 acts as a biological surfactant to disperse mitotic chromosomes. Nature. 2016; 535: 308-12.

35. Silva DC, Goncalves AK, Cobucci RN, Mendonca RC, Lima PH, Cavalcanti GJ. Immunohistochemical expression of p16, Ki-67 and p53 in cervical lesions - A systematic review. Pathol Res Pract. 2017; 213: 723-9.

36. Carozzi F, Gillio-Tos A, Confortini M, Del Mistro A, Sani C, De Marco L, et al. Risk of high-grade cervical intraepithelial neoplasia during follow-up in HPV-positive women according to baseline p16-INK4A results: a prospective analysis of a nested substudy of the NTCC randomised controlled trial. Lancet Oncol. 2013; 14: 168-76.

37. Yu LL, Chen $\mathrm{W}$, Lei XQ, Qin $\mathrm{Y}, \mathrm{Wu} Z \mathrm{ZN}$, Pan QJ, et al. Evaluation of p16/Ki-67 dual staining in detection of cervical precancer and cancers: a multicenter study in China. Oncotarget. 2016; 7: 21181-9.

38. Dona MG, Vocaturo A, Giuliani M, Ronchetti L, Rollo F, Pescarmona E, et al. p16/Ki-67 dual staining in cervico-vaginal cytology: correlation with histology, Human Papillomavirus detection and genotyping in women undergoing colposcopy. Gynecol Oncol. 2012; 126: 198-202.

39. Uijterwaal MH, Witte BI, Van Kemenade FJ, Rijkaart D, Ridder R, Berkhof J, et al. Triaging borderline/mild dyskaryotic Pap cytology with p16/Ki-67 dual-stained cytology testing: cross-sectional and longitudinal outcome study. Br J Cancer. 2014; 110: 1579-86.

40. Rossi P, Borghi L, Ferro R, Mencarelli R. A population of 1136 HPV DNA-HR positive women: expression of p16(INK4a)/Ki67 Dual-Stain Cytology and cytological diagnosis. Histological correlations and cytological follow up. Pathologica. 2015; 107: 185-91.

41. Wentzensen N, Fetterman B, Castle PE, Schiffman M, Wood SN, Stiemerling E, et al. p16/Ki-67 Dual Stain Cytology for Detection of Cervical Precancer in HPV-Positive Women. J Natl Cancer Inst. 2015; 107: djv257.

42. Ordi J, Sagasta A, Munmany M, Rodriguez-Carunchio L, Torne A, del Pino M. Usefulness of p16/Ki67 immunostaining in the triage of women referred to colposcopy. Cancer Cytopathol. 2014; 122: 227-35.

43. Wright TC, Jr, Behrens CM, Ranger-Moore J, Rehm S, Sharma A, Stoler MH, et al. Triaging HPV-positive women with p16/Ki-67 dual-stained cytology: Results from a sub-study nested into the ATHENA trial. Gynecol Oncol. 2017; 144: 51-6.

44. Petry KU, Menton S, Menton M, van Loenen-Frosch F, de Carvalho Gomes $\mathrm{H}$, Holz B, et al. Inclusion of HPV testing in routine cervical cancer screening for women above 29 years in Germany: results for 8466 patients. Br J Cancer. 2003; 88: 1570-7.

45. Castle PE, Fetterman B, Poitras N, Lorey T, Shaber R, Kinney W. Five-year experience of human papillomavirus DNA and Papanicolaou test cotesting. Obstet Gynecol. 2009; 113: 595-600.

46. Thrall MJ, Russell DK, Facik MS, Yao JL, Warner JN, Bonfiglio TA, et al. High-risk HPV testing in women 30 years or older with negative Papanicolaou tests: initial clinical experience with 18-month follow-up. Am J Clin Pathol. 2010; 133: 894-8.

47. Uijterwaal MH, Polman NJ, Witte BI, van Kemenade FJ, Rijkaart D, Berkhof J, et al. Triaging HPV-positive women with normal cytology by $\mathrm{p} 16 / \mathrm{Ki}-67$ dual-stained cytology testing: baseline and longitudinal data. Int J Cancer. 2015; 136: 2361-8.

48. Bergeron C, Ikenberg H, Sideri M, Denton K, Bogers J, Schmidt D, et al. Prospective evaluation of p16/Ki-67 dual-stained cytology for managing women with abnormal Papanicolaou cytology: PALMS study results. Cancer Cytopathol. 2015; 123: 373-81.

49. White C, Bakhiet S, Bates M, Keegan H, Pilkington L, Ruttle C, et al. Triage of LSIL/ASC-US with p16/Ki-67 dual staining and human papillomavirus testing: a 2-year prospective study. Cytopathology. 2016; 27: 269-76.

50. Kocken M, Helmerhorst TJ, Berkhof J, Louwers JA, Nobbenhuis MA, Bais AG, et al. Risk of recurrent high-grade cervical intraepithelial neoplasia after successful treatment: a long-term multi-cohort study. Lancet Oncol. 2011; 12: $441-50$

51. Melnikow J, McGahan C, Sawaya GF, Ehlen T, Coldman A. Cervical intraepithelial neoplasia outcomes after treatment: long-term follow-up from the British Columbia Cohort Study. J Natl Cancer Inst. 2009; 101: 721-8.

52. Arbyn M, Ronco G, Anttila A, Meijer CJLM, Poljak M, Ogilvie G, et al. Evidence Regarding Human Papillomavirus Testing in Secondary Prevention of Cervical Cancer. Vaccine. 2012; 30: F88-F99.

53. Polman NJ, Uijterwaal MH, Witte BI, Berkhof J, van Kemenade FJ, Spruijt JWM, et al. Good performance of p16/ki-67 dual-stained cytology for surveillance of women treated for high-grade CIN. Int J Cancer. 2017; 140; 423-30.

54. Nucci MR. Symposium part III: tumor-like glandular lesions of the uterine cervix. Int J Gynecol Pathol. 2002; 21: 347-59.

55. Ravarino A, Nemolato S, Macciocu E, Fraschini M, Senes G, Faa G, et al. CINtec PLUS immunocytochemistry as a tool for the cytologic diagnosis of glandular lesions of the cervix uteri. Am J Clin Pathol. 2012; 138: 652-6.

56. Singh M, Mockler D, Akalin A, Burke S, Shroyer A, Shroyer KR. Immunocytochemical colocalization of P16(INK4a) and Ki-67 predicts CIN2/3 and AIS/adenocarcinoma. Cancer Cytopathol. 2012; 120: 26-34.

57. Grabe N, Lahrmann B, Pommerencke T, von Knebel Doeberitz M, Reuschenbach M, Wentzensen N. A virtual microscopy system to scan, evaluate and archive biomarker enhanced cervical cytology slides. Cell Oncol. 2010; 32: 109-19.

58. Lahrmann B, Valous NA, Eisenmann U, Wentzensen N, Grabe N. Semantic focusing allows fully automated single-layer slide scanning of cervical cytology slides. PLoS One. 2013; 8: e61441. 